Journal for ImmunoTherapy of Cancer

\section{Effect of cyclo-oxygenase inhibitor use during checkpoint blockade immunotherapy in patients with metastatic melanoma and non-small cell lung cancer}

To cite: Wang S-J, Khullar K, Kim S, et al. Effect of cyclooxygenase inhibitor use during checkpoint blockade immunotherapy in patients with metastatic melanoma and nonsmall cell lung cancer. Journal for ImmunoTherapy of Cancer 2020;8:e000889. doi:10.1136/ jitc-2020-000889

JMM and SKJ are joint senior authors.

Accepted 05 August 2020
Check for updates

(c) Author(s) (or their employer(s)) 2020. Re-use permitted under CC BY-NC. No commercial re-use. See rights and permissions. Published by BMJ.

For numbered affiliations see end of article.

Correspondence to Dr Salma K Jabbour; jabbousk@cinj.rutgers.edu

\section{ABSTRACT}

Background Immune checkpoint inhibitors (ICls) improve survival outcomes in metastatic melanoma and non-small cell lung cancer (NSCLC). Preclinical evidence suggests that overexpression of cyclo-oxygenase-2 (COX2) in tumors facilitates immune evasion through prostaglandin E2 production and that COX inhibition synergizes with ICls to promote antitumor T-cell activation. This study investigates whether concurrent COX inhibitor (COXi) use during $\mathrm{ICl}$ treatment compared with $\mathrm{ICI}$ alone is associated with improved time-to-progression (TTP), objective response rate (ORR) and overall survival (OS) in patients with metastatic melanoma and NSCLC.

Methods We retrospectively reviewed 90 metastatic melanoma and 37 metastatic NSCLC patients, treated with ICI between 2011 and 2019. Differences in TTP and OS by $\mathrm{ICl}+\mathrm{COXi}$ versus $\mathrm{ICl}$ alone were compared using KaplanMeier and Cox regression. Interaction between $\mathrm{ICl}+\mathrm{COXi}$ versus ICl alone and pretreatment neutrophil-lymphocyte ratio (NLR) was examined. Independent radiology review per Response Evaluation Criteria in Solid Tumors (RECIST) version 1.1 was performed.

Results For patients with melanoma, median TTP was significantly prolonged in $\mathrm{ICl}+\mathrm{COXi}$ versus $\mathrm{ICl}$ alone (245 vs 100.5 days, $\mathrm{p}=0.002$ ). On multivariate analysis, $\mathrm{ICl}+\mathrm{COXi}$ associated with increased TTP (HR 0.36, 95\% $\mathrm{Cl} 0.2$ to $0.66, p=0.001$ ), adjusted for age, pretreatment NLR, and gender. For NSCLC patients, ICl+COXi also associated with increased TTP compared with ICl alone on multivariate analysis (HR $0.45 ; 95 \% \mathrm{Cl} 0.21$ to $0.97 ; \mathrm{p}=0.042$ ) adjusted for age. ORR at 6 months was significantly higher in patients who received $\mathrm{ICl}+\mathrm{COXi}$ compared with $\mathrm{ICI}$ alone in both melanoma ( $58.6 \%$ vs $19.2 \%, p=0.0005)$ and NSCLC $(73.7 \%$ vs $33.3 \%, p=0.036)$ cohorts. In the melanoma cohort, high pretreatment NLR ( $>5)$ associated with decreased TTP (HR 3.21, 95\% Cl 1.64 to $6.3 ; p=0.0007$ ); however, $\mathrm{ICl}+\mathrm{COXi}$ significantly associated with increased TTP in high NLR (>5) patients (HR 0.08, 95\% Cl 0.03 to $0.25)$, but not in low NLR $(\leq 5)$ patients (HR $0.65,95 \% \mathrm{Cl}$ 0.32 to 1.32). Similar outcomes were found in an adjusted melanoma cohort after RECIST review.
Conclusions Our study suggests that COXi use concurrently with ICl significantly associated with longer TTP and improved ORR at 6 months in patients with metastatic melanoma and NSCLC compared with ICI alone. Furthermore, COXi use appears to reverse the negative prognostic effect of a high NLR by prolonging TTP in patients with melanoma.

\section{BACKGROUND}

Immune checkpoint inhibitors (ICIs) blocking the cytotoxic T-lymphocyteassociated protein 4 (CTLA-4) and programmed cell death protein/ligand 1 (PD-1/PD-L1) improve the survival and treatment response rates in both melanoma and non-small cell lung cancer (NSCLC) in the setting of metastatic disease. ${ }^{1-8}$ However, despite the remarkable response of ICIs in a subset of patients, approximately $50 \%-80 \%$ of patients in these trials do not respond or develop adaptive resistance to therapy. ${ }^{1-8}$

Cyclo-oxygenase-2 (COX2) gene is frequently overexpressed in melanoma, NSCLC and other cancers, and the consequential production of prostaglandin E2 $\left(\mathrm{PGE}_{2}\right)$ associates with poor prognoses. ${ }^{9} 10$ The pro-tumoral effect of the COX2-PGE axis links to tumor cell proliferation and differentiation, chronic inflammation, angiogenesis, and tumor immunosuppression. ${ }^{9}$ Also, the inhibition of COX2 activity using aspirin reduces the incidence, mortality, and risk of metastasis in colorectal cancer. ${ }^{11-15}$

Preclinical studies demonstrate that the COX2 pathway contributes to tumor immune evasion in melanoma models. COX2 expression allows tumor cells to evade type I interferon-mediated immunity, and the combination of COX and PD-1 inhibition 
results in remarkable therapeutic synergy in murine melanoma models. ${ }^{16} \mathrm{~A}$ follow-up study from the same group reveals that tumor-derived $\mathrm{PGE}_{2}$ impairs the ability of natural killer cells to recruit dendritic cells intratumorally, posing another mechanism of immune checkpoint exploited by tumor cells. ${ }^{17}$ Analysis of The Cancer Genome Atlas database positively correlates a COXdeficient immune molecular signature with improved survival outcomes across several tumor types, including melanoma and NSCLC, underscoring the translational value of this mechanistic discovery. ${ }^{17}$

Based on these data, we hypothesize that the use of COX inhibitors (COXi) could improve the efficacy of ICI as measured by time-to-progression (TTP), objective response rate (ORR) and overall survival (OS) after initiating ICI therapy in a cohort of patients with metastatic melanoma and NSCLC that were treated only with ICI.

\section{METHODS \\ Patients}

Patients with metastatic melanoma $(\mathrm{n}=90)$ and NSCLC $(\mathrm{n}=37)$ who were treated with their first-courses of immunotherapy after their metastatic diagnoses with either single-agent (ipilimumab, pembrolizumab, nivolumab, atezolizumab, avelumab, or cemiplimab) or dual-agent (ipilimumab/nivolumab) ICI, with treatment initiation between May 2011 to February 2019 were identified at Rutgers Cancer Institute of New Jersey. Inclusion criteria were: (1) Eastern Cooperative Oncology Group (ECOG) performance status $\leq 2$; (2) treatment using ICI alone, without combination with other forms of immunotherapy (ie, oncolytic virus or interleukins) or chemotherapy; (3) received $\geq 2$ cycles of ICI; and (4) a minimum follow-up of 2 months after receiving their last cycle of ICI or until progressive disease (PD). COXi use was defined as having received COXi (aspirin, non-steroidal anti-inflammatory drugs (NSAIDs), or selective COX2 inhibitors) during, at least, the initial two cycles of ICI. Clinicopathological characteristics, including age, gender, ECOG performance status, pretreatment neutrophil-to-lymphocyte ratio (NLR) from peripheral blood within 1 week before starting ICI, tumor mutation burden, and steroid use during treatment, were collected. Additionally, we collected BRAF mutational status for patients with melanoma and histology for NSCLC patients.

Clinical PD was determined by treating physician based on imaging (CT, positron emission tomography, or MRI) or physical exam as documented in the medical records. Imaging review by radiologists (SHC and JLN) using Response Evaluation Criteria in Solid Tumors (RECIST) version 1.1 was independently performed for validation in the melanoma cohort. Of the 90 patients with melanoma, 72 patients had complete imaging data for assessment. Clinical and radiographic assessments were congruent in $62.5 \%(\mathrm{n}=45)$ who were assessed as PD and 29.2\% $(\mathrm{n}=21)$ who were assessed as non-PD (stable disease, partial response, or complete response). Only $4.2 \%(n=3)$ of patients had radiographic PD at earlier time points than clinically declared and $4.2 \% \quad(\mathrm{n}=3)$ had radiographic non-PD despite declared clinical PD. A modified melanoma cohort $(\mathrm{n}=69)$ for validation purpose included the 66 patients with radiographic assessments congruent with clinical management, as well as the three patients with time of PD adjusted to earlier dates after imaging review.

\section{Statistical analysis}

Patient characteristics were described according to the status of COXi use during first-course ICI and compared with Fisher's exact test for categorical variables, or Wilcoxon rank sum test for continuous variables. TTP was defined as the time from ICI initiation to clinical PD. ORR at 6 months was defined as the proportion of patients with complete or partial response after 6 months of initiating ICI; patients were ineligible for ORR calculation if they had treatment response but had $<6$ months of follow-up. OS was defined as the time from ICI initiation to death of any cause. Patients without an event were censored at the date of last known follow-up. Survival curves were estimated using the Kaplan-Meier method and compared using log-rank statistics. Univariate HRs were estimated for various clinicopathological characteristics using Cox proportional hazard regression model. Proportionality hazard (PH) assumption was evaluated. The stratified Cox proportional hazard model was also employed for covariates not satisfying the $\mathrm{PH}$ assumptions for multivariate setting. Predictors which were statistically significant in univariate setting were included in multivariable model. Due to limited data available, tumor mutation burden could not be included in the Cox regression model. Interaction effect of COXi use and NLR on TTP was also tested in multivariable model. Fisher's exact test was employed to compare the ORR at 6 months. All statistical analyses were performed using SAS software V.9.4.

\section{RESULTS \\ Melanoma cohort}

Descriptive statistics

Our analysis examined 90 patients with metastatic melanoma who met the inclusion criteria. The median follow-up time was 3.56 years (95\% CI 2.52 to 3.98). For patients who received ICI alone $(n=58,64.4 \%)$, the majority of patients were male $(62.1 \%)$, the median age was 60 years (range $41-89), 34 \%$ of patients $(n=18)$ had high pretreatment NLR $(>5)$, and $62.1 \%$ of patients did not have a BRAF mutation (table 1). For patients who received ICI with concurrent COXi $(n=32,35.6 \%)$, the majority of patients were men $(68.7 \%)$, the median age was 69 years (range $34-91), 32.3 \%$ of patients $(n=10)$ had high pretreatment NLR $(>5)$, and $65.6 \%$ of patients did not have a BRAF mutation. The reasons for COXi use were cardiovascular prevention $(78.1 \%, \mathrm{n}=25)$, chronic pain/arthritis $(9.4 \%, \mathrm{n}=3)$, cancer-related pain $(9.4 \%$, $\mathrm{n}=3$ ) and unknown $(3.1 \%, \mathrm{n}=1)$. Median age was significantly higher in patients with melanoma who received 







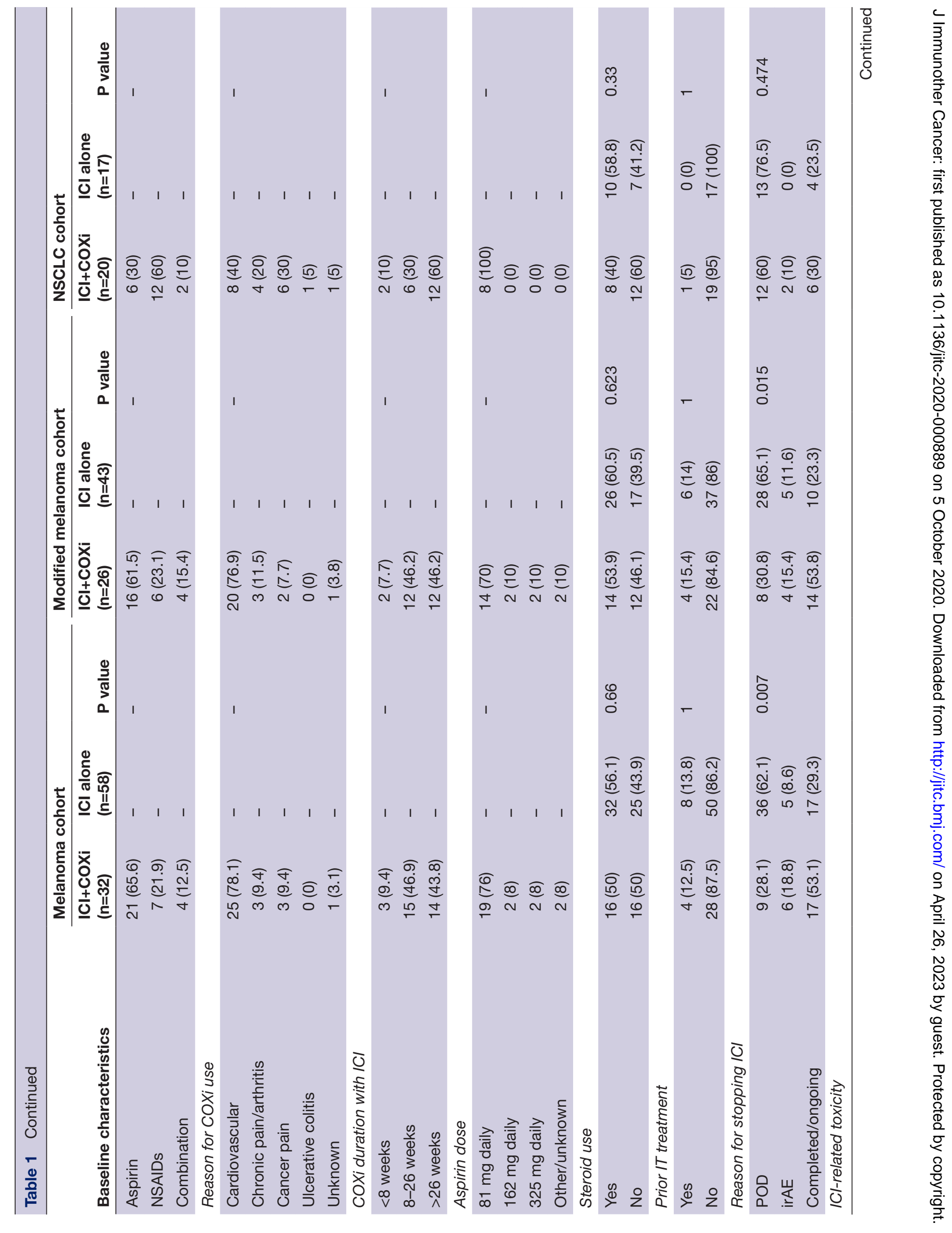




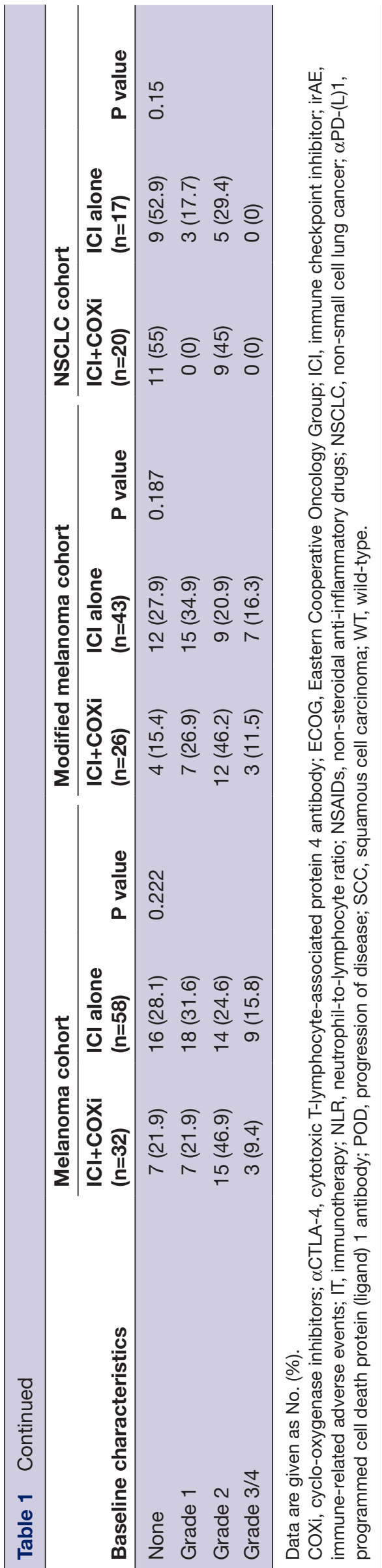

ICI+COXi versus those who received ICI alone $(\mathrm{p}=0.006)$. Otherwise, distributions of other covariates were not significantly different between the groups (table 1).

\section{TTP, ORR, and OS analysis by treatment received}

In the melanoma cohort, the median TTP was significantly prolonged in patients who received ICI+COXi (245 days) versus patients who received ICI alone (100.5 days, $\mathrm{p}=0.002$ ) (figure 1A). On univariate analysis, ICI+COXi significantly associated with an increased TTP (HR 0.48, $95 \%$ CI 0.29 to $0.77 ; p=0.003$ ), while effect of age, gender, presence of a BRAF mutation, steroid use, and type of COXi on TTP were not (table 2). On multivariate analysis, treatment with ICI+COXi (HR 0.36, 95\% CI 0.20 to $0.66 ; \mathrm{p}=0.0006$ ) and female gender (HR $0.34,95 \%$ CI 0.18 to $0.66 ; p=0.001$ ) remained associated with longer TTP in the melanoma cohort.

ORR at 6 months for patients with melanoma were significantly higher in patients who received ICI+COXi $(58.6 \%)$ compared with those who received ICI alone $(19.2 \%, \mathrm{p}=0.0005)$.

Median OS for patients with melanoma did not differ between those who received ICI+COXi (2.12 years, 95\% CI 1.23 to 2.52) compared with those who received ICI alone ( 1.84 years, $95 \%$ CI 1.32 to $2.53 ; \mathrm{p}=0.91$ ) (figure $2 \mathrm{~A}$ and table 2).

Interaction effects with treatment received Pretreatment NLR

High pretreatment NLR $>5$ associated significantly with decreased TTP on both univariate (HR 1.76, 95\% CI 1.08 to 2.87; $\mathrm{p}=0.024$ ) and multivariate analyses (HR 3.21, 95\% CI 1.64 to $6.3 ; \mathrm{p}=0.0007$ ) in patients with melanoma (table 2). Moreover, we detected a significant interaction effect between treatment received (ICI+COXi vs ICI alone) and pretreatment NLR (effect estimate -2.09 , $\mathrm{SE}=0.67, \mathrm{p}=0.002$ ), adjusted for gender and age (table 3 ). In patients with high pretreatment NLR $>5$, ICI+COXi prolonged TTP compared with ICI alone (HR 0.08, 95\% CI 0.03 to 0.25 ) (table 3 and figure $3 \mathrm{~A}$ ). On the contrary, ICI+COXi was not significantly associated with TTP in patients with low pretreatment $\mathrm{NLR} \leq 5$ when compared with ICI alone (HR $0.65,95 \%$ CI 0.32 to 1.32 ).

To confirm our results, we performed additional analyses using pretreatment NLR as a continuous logarithmic variable. First, multivariable analysis demonstrated that an increase in continuous log-scale NLR is associated with shorter TTP (HR 2.11, 95\% CI 1.3 to 3.41; $\mathrm{p}=0.002$ ) (table 2). Second, the interaction between log-scale NLR and ICI+COXi versus ICI alone remained significant (effect estimate $-1.53, \mathrm{p}=0.004$ ). For patients who received ICI alone, incremental increase in log-scale NLR was associated with incremental shorter TTP (HR 3.35, 95\% CI 1.85 to 6.05$)$. On the contrary, for patients who received ICI+COXi, incremental increase in log-scale NLR was not significantly associated with TTP (HR 0.72 , $95 \%$ CI 0.31 to 1.7$)$. 
(A)

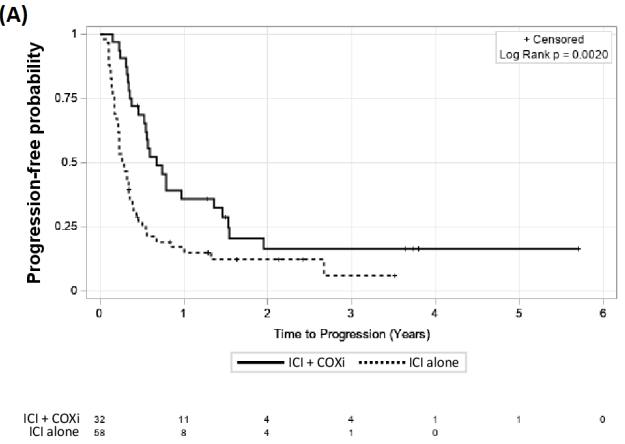

(C)

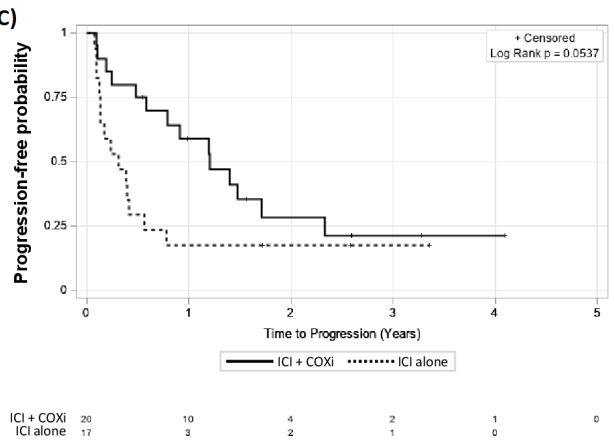

(B)

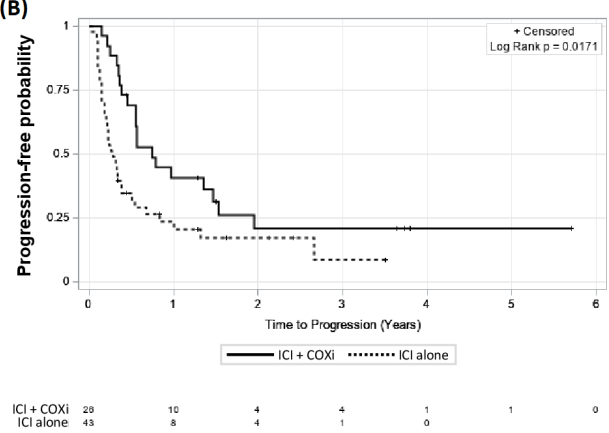

Interaction between the type of ICI and COXi use on TTP was also analyzed. Among patients who were treated with CTLA-4 inhibitor monotherapy, those that received ICI+COXi combination were found to have a significantly longer TTP compared with those that received ICI alone (HR 0.33, 95\% CI 0.13 to 0.82) (table 3). However, ICI+COXi combination did not significantly impact TTP in patients treated with PD-(L) 1 inhibitors (HR 0.62, $95 \%$ CI 0.3 to 1.26 ) or dual CTLA-4/PD-1 combination blockade (HR $0.47,95 \%$ CI 0.13 to 1.77 ).

\section{Modified melanoma cohort after RECIST assessment}

To verify our results, we performed RECIST assessment of patients' radiographic studies and included 69 evaluable patients in our modified cohort (see the Methods and table 1) which resulted in similar outcomes. In patients who received ICI+COXi, median TTP was 273 days (95\% CI 166 to 560) compared with 105 days $(95 \%$ CI 65 to 141) in those who received ICI alone (figure 1B). On both univariate and multivariate analysis, ICI+COXi (HR 0.51 (0.29-0.9), p=0.017, and HR 0.31 (0.15-0.64), $\mathrm{p}=0.002$, respectively) and pretreatment NLR (HR 1.93 (1.14-3.44), $\mathrm{p}=0.015$, and HR 3.33 (1.56-7.13), $\mathrm{p}=0.002$, respectively) significantly associated with TTP (table 2). In addition, interaction effect between pretreatment NLR and ICI+COXi versus ICI alone on TTP remained significant (effect estimate-2.42; SE 0.82; p=0.003) (table 3 and figure 3B). Finally, the subset of patients who received CTLA-4 inhibitor monotherapy significantly benefited from the combination of ICI+COXi (HR 0.21, 95\% CI 0.06 to 0.79 ) (table 3 ).

\section{NSCLC cohort}

Descriptive statistics

To examine whether the results from the melanoma cohort could be applicable to another cancer type, we examined an additional 37 patients with metastatic NSCLC who met the inclusion criteria. The median follow-up time was 2.89 years (95\% CI 1.82 to 3.58 ). Twenty of these patients received ICI+COXi $(54.1 \%)$, while 17 patients received ICI alone $(45.9 \%)$. For patients who received ICI alone, most patients $(70.6 \%)$ were women, the median age was 67 (range 50-82), $62.5 \%$ of patients $(n=10)$ had high pretreatment NLR $(>5)$, and $70.6 \%$ of patients had adenocarcinoma (table 1). For patients who received ICI+COXi, most patients $(80 \%)$ were women, the median age was 65.5 years (range $55-86), 25 \%$ patients $(n=5)$ had high pretreatment NLR $(\mathrm{NLR}>5)$, and $80 \%$ of patients had adenocarcinoma. The reasons for $\mathrm{COXi}$ use were cardiovascular prevention (40\%, $\mathrm{n}=8)$, chronic pain/arthritis $(20 \%, \mathrm{n}=4)$, cancerrelated pain $(30 \%, \mathrm{n}=6)$, ulcerative colitis $(5 \%, \mathrm{n}=1)$ and unknown $(5 \%, \mathrm{n}=1)$. The proportion of NSCLC patients with high pretreatment NLR was significantly greater in those that received ICI alone compared with those who received ICI+COXi $(\mathrm{p}=0.041)$. Otherwise, distributions of other covariates were not significantly different between two groups (table 1). 


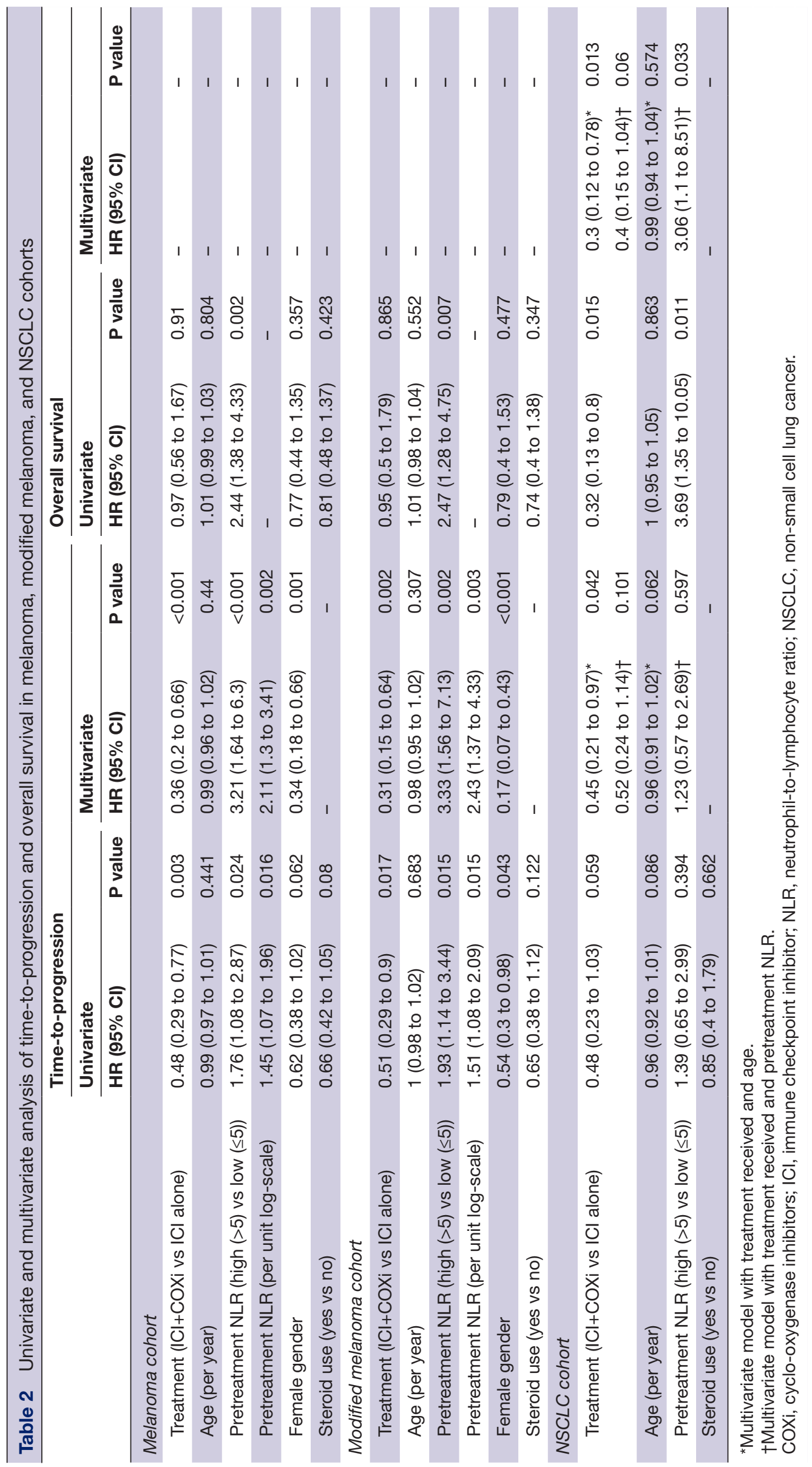



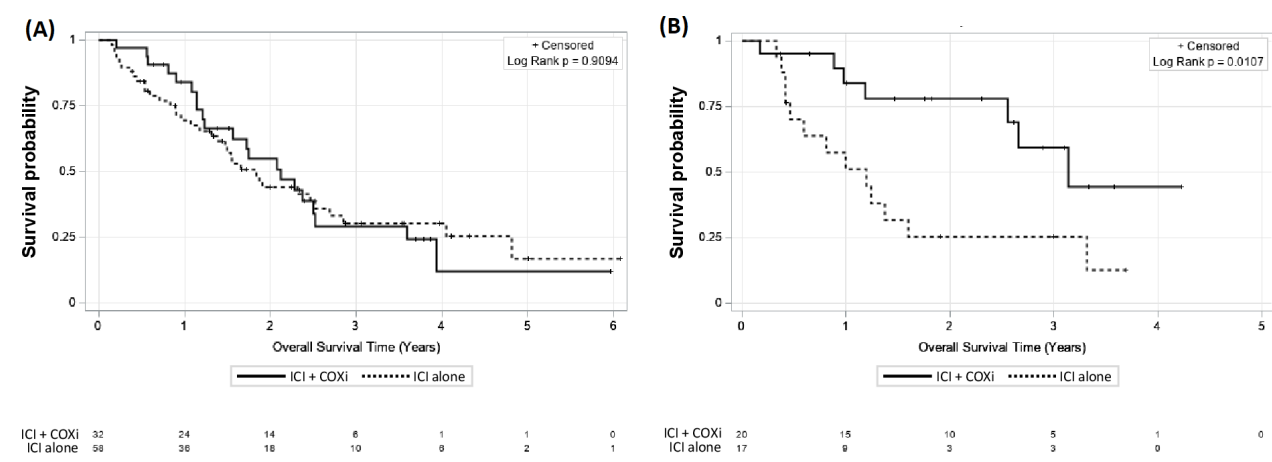

Figure 2 Effect of COXi use on overall survival. Kaplan-Meier curves for overall survival in (A) metastatic melanoma cohort $(n=90)$, and (B) metastatic non-small cell lung cancer cohort $(n=37)$. COXi, cyclo-oxygenase inhibitors; $I C l$, immune checkpoint inhibitor.

TTP, ORR, and OS analysis by treatment received

In the NSCLC cohort, ICI+COXi did not significantly increase median TTP compared with those receiving ICI alone, though there was likely a clinical benefit (440 vs 113 days, $\mathrm{p}=0.054$ ) (figure 1C). On univariate analysis, ICI+COXi was not associated with a significant increase in TTP, though there was again a favorable trend (HR 0.48 , $95 \%$ CI 0.23 to $1.03 ; p=0.059$ ) (table 2 ). On multivariate analysis using a model including treatment received and age, ICI+COXi was associated with a significantly increased TTP (HR 0.45, 95\% CI 0.21 to 0.97; $\mathrm{p}=0.042$ ) (table 2).

ORR at 6 months for NSCLC patients were significantly higher in patients who received ICI+COXi (73.7\%) compared with those who received ICI alone $(33.3 \%$, $\mathrm{p}=0.036$ ).

The median OS for NSCLC patients was significantly increased in patients who received ICI+COXi (3.14 years, 95\% CI 2.56 to NA) compared with those who received ICI alone (1.19 years, 95\% CI 0.42 to $1.59 ; \mathrm{p}=0.012$ ) (figure 2B). On univariate analysis, ICI+COXi associated with improved OS (HR $0.32,95 \%$ CI 0.13 to 0.8 ;

Table 3 Interaction effect between COXi use and pretreatment NLR or type of ICI

\begin{tabular}{|c|c|c|}
\hline & \multicolumn{2}{|c|}{$\mathrm{ICl}+\mathrm{COXi}$ versus $\mathrm{ICI}$ alone } \\
\hline & Melanoma cohort & $\begin{array}{l}\text { Modified melanoma } \\
\text { cohort }\end{array}$ \\
\hline & HR (95\% Cl) & HR (95\% Cl) \\
\hline \multicolumn{3}{|l|}{ Pretreatment NLR } \\
\hline High (NLR>5) & $0.08(0.03$ to 0.25$)$ & 0.06 (0.02 to 0.24$)$ \\
\hline Low (NLR $\leq 5)$ & 0.65 (0.32 to 1.32$)$ & 0.7 (0.3 to 1.62$)$ \\
\hline \multicolumn{3}{|l|}{ Type of ICI } \\
\hline$\alpha \mathrm{PD}-(\mathrm{L}) 1$ & 0.62 (0.3 to 1.26$)$ & 0.6 (0.27 to 1.34$)$ \\
\hline$\alpha C T L A-4$ & 0.33 (0.13 to 0.82$)$ & 0.21 (0.06 to 0.79$)$ \\
\hline$\alpha$ CTLA-4+ $+\alpha$ PD- 1 & $0.47(0.13$ to 1.77$)$ & $0.55(0.14$ to 2.14$)$ \\
\hline
\end{tabular}

COXi, cyclo-oxygenase inhibitors; $\alpha \mathrm{CTLA}-4$, cytotoxic Tlymphocyte-associated protein 4 antibody; ICl, immune checkpoint inhibitor; NLR, neutrophil-to-lymphocyte ratio; $\alpha P D$ (L)1, programmed cell death protein (ligand) 1 antibody. $\mathrm{p}=0.015$ ). In a multivariate analysis model including treatment received and age, ICI+COXi was associated with increased OS (HR $0.3,95 \%$ CI 0.12 to $0.78 ; \mathrm{p}=0.013$ ). However, using a multivariate model with treatment received and pretreatment NLR, ICI+COXi was associated with trending OS benefit (HR $0.4,95 \%$ CI 0.15 to $1.04 ; \mathrm{p}=0.06)$.

Effect of pretreatment NLR on TTP and OS

High versus low pretreatment NLR did not significantly impact TTP on either univariate or multivariate analyses. In contrast, high pretreatment NLR was associated with decreased OS on univariate analysis (HR 3.69, 95\% CI 1.35 to $10.05 ; \mathrm{p}=0.011$ ) and in a multivariate model with treatment received and pretreatment NLR (HR 3.06, 95\% CI 1.1 to $8.51 ; \mathrm{p}=0.033$ ) (table 2 ).

\section{DISCUSSION}

Our study demonstrated that, among patients with metastatic melanoma and NSCLC, COXi use during the first courses of ICI (ICI+COXi) was associated with longer TTP. ORR at 6 months were significantly higher in patients who received ICI+COXi compared with those who received ICI alone in both melanoma and NSCLC cohorts. While COXi use had no effect on OS in the melanoma cohort, it associated with improved OS in patients with metastatic NSCLC. Furthermore, we detected a significant interaction between COXi use and pretreatment NLR on TTP in the melanoma cohort, with ICI+COXi seeming to negate the negative predictive value of high pretreatment NLR. Subset analysis by the type of ICI received revealed a significant benefit of COXi in prolonging TTP in patients with melanoma treated with CTLA-4 inhibitor. Finally, we validated our results using a modified melanoma cohort after RECIST assessment and showed similar outcomes.

COX2-mediated $\mathrm{PGE}_{2}$ synthesis suppresses antitumor immunity through diverse mechanisms, including inhibition of myeloid cell activation, decreased natural killer and dendritic cell recruitment, regulatory T-cell induction, and decreased proliferation and effector function of CD4/8 T-cells, and COX inhibition has been shown to overcome tumor immune evasion and synergize with 

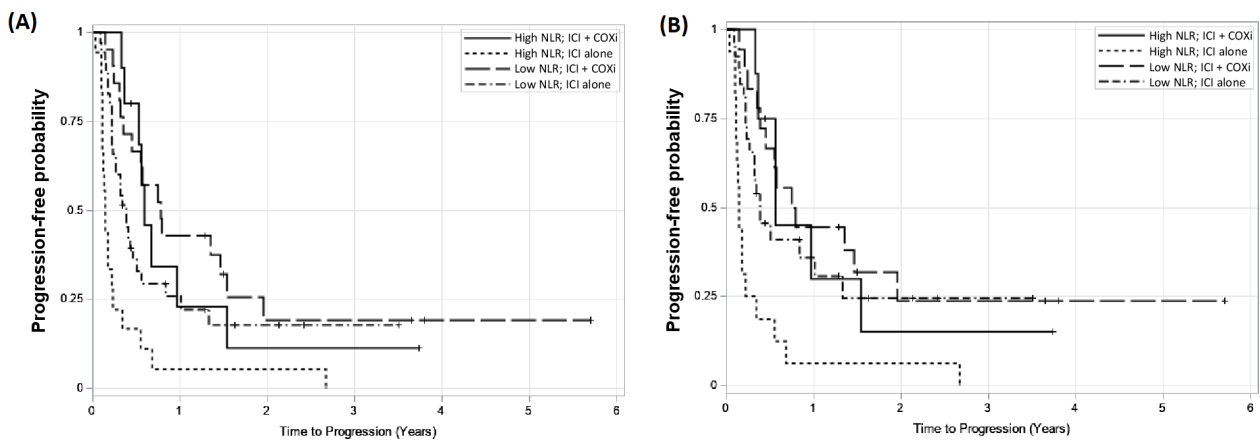

Figure 3 Interaction effect between COXi use and pretreatment NLR. Kaplan-Meier curves for time-to-progression depicting the interaction between high versus low pretreatment NLR and COXi use in (A) melanoma cohort, and (B) modified melanoma cohort after Response Evaluation Criteria in Solid Tumors assessment. COXi, cyclo-oxygenase inhibitors; ICI, immune checkpoint inhibitor; NLR, neutrophil-to-lymphocyte ratio.

checkpoint blockade in animal models. ${ }^{1617}$ However, there is a paucity of clinical evidence to date that demonstrates the potential therapeutic benefit of concurrent COXi use with ICIs. Our results demonstrate the potential benefit of COXi use in prolonging TTP with ICI, which support our hypothesis that concomitant COXi use may potentiate the efficacy of ICIs through inhibiting COX2-mediated immunosuppression. Although not available in this study, serum PGE2 or other related immune biomarkers for assessing COX2 activity may serve as important biological correlatives for future prospective studies.

Clinical evidence also indirectly supports the potential synergy between COXi and ICIs. A secondary analysis of two prospective cohort studies correlated improved colorectal cancer survival from aspirin use with low tumorous CD274 (PD-L1) expression. ${ }^{13}$ Differential effect of CD274 status on the antitumor efficacy of aspirin underscores the potential multiplicity of immunosuppressive mechanisms deployed by tumors. Furthermore, greater cancer survival with aspirin use in CD274-low tumors suggests that blockade of the PD-1/PD-L1 axis in CD274-high tumors may synergize with the immunomodulatory effects of COX inhibition. On the other hand, Botti et al revealed that COX2 expression positively correlated with PD-L1 expression in melanoma tumor samples, implying that the synergy from concomitant COX inhibition may potentially be more pronounced in patients with higher predicted response to PD-1/PD-L1 checkpoint blockade. ${ }^{18}$ The elucidated mechanism of action suggests that COX inhibition leads to antitumor dendritic cell activation, which occurs upstream of checkpoint blockade. ${ }^{16}{ }^{17}$ As such, design of future prospective trials investigating the synergy between COXi and ICI would likely gain from testing concomitant COXi use with ICI, which is reflected in our study methodology of analyzing patients who have at least received COXi during their first two cycles of ICI.

A recent exploratory analysis of the REMAGUS02 trial reported survival detriment with COX2-inhibitor celecoxib use in PTGS2 (COX2)-low breast cancer patients, but not in PTGS2-high patients, who received neoadjuvant docetaxel chemotherapy. ${ }^{19}$ There was significant interaction between celecoxib use and PTGS2 expression, suggesting that both the deleterious effect of celecoxib use with chemotherapy and the therapeutic benefit of COX inhibition in PTGS2-high patients are at play. Several other prospective trials also reported a lack of benefit or association with poorer outcomes with COX2inhibitor use in patients receiving chemotherapy. ${ }^{2021}$ This is in contrast with our results, but given the immunosuppressive function of PTGS2 overexpression, one would expect greater therapeutic synergy of COXi with ICIs, compared with with chemotherapy. In addition, most patients in our cohort used aspirin and NSAIDs, rather than selective COX2-inhibitors.

Our analysis demonstrated that low pretreatment NLR associated with response to ICI in the melanoma cohort, with high NLR $(>5)$ correlating to decreased TTP and OS. Remarkably, our results also showed that the detrimental effect of high pretreatment NLR on TTP was reversed or negated in patients with melanoma who received concurrent COXi with ICIs; the associated benefit from concomitant COXi use was greater in patients with high NLR than in those with low NLR. Emerging evidence indicates that pretreatment NLR is a prognostic marker for a wide range of malignancies, with NLR values of $>4$ or $>5$ being associated with worsening OS and/or diseasefree survival. ${ }^{22-24}$ Results from retrospective analyses of patients with melanoma and NSCLC were also suggestive of NLR having potential predictive value in the response to immunotherapy. ${ }^{25}{ }^{26}$ To date, the underlying biological mechanism of NLR as a novel biomarker is unclear and efforts are underway to elucidate this. Likely an oversimplification, NLR represents the balance between the immunosuppressive protumor neutrophil population and the adaptive antitumor immunity conferred by lymphocytes. ${ }^{23} 27$ This implies that COX inhibition may potentially mitigate systemic immune tolerance characterized by high NLR. Although there was no association between NLR and TTP in the NSCLC cohort, the association between NLR and OS was significant. Future prospective studies are warranted to validate the effect of COXi on the predictive value of NLR in patients receiving ICIs. 
Stratification of patients with melanoma by the ICI types used revealed a significant increase in TTP associated with COXi use in patients who received anti-CTLA-4 (ipilimumab) monotherapy. While COXi use was not significantly associated with an increase in TTP in the subgroups of patients who received PD-1/PD-L1 blockade or CTLA-4/PD-1 combination, there were trends that skewed largely toward favorable outcomes with COXi use. The lack of statistical significance is likely due to small sample sizes in subgroup analysis. However, our results indicate that the benefit of COX inhibition may be more pronounced when combined with anti-CTLA-4 blockade. It is possible that greater synergy exists between CTLA-4 blockade and COXi, given that both agents target the initial cascades of T cell priming by dendritic cells. ${ }^{16} 1728$

Although data for concurrent COXi use with ICIs must be obtained from real-world studies such as this one, given the lack of prospective evidence, there are several limitations to consider. Outcomes of this study were assessed retrospectively, and therefore, are subject to potential bias and confounding from unmeasured variables. However, we were able to validate our results through objective response assessment by radiologists per the RECIST criteria. The sample size of this study is relatively small, given that this is a single institution study with multiple exclusion criteria in patient selection aimed to minimize confounding bias. As such, we were unable to demonstrate statistical significance in all the endpoints of our subgroup analysis, although many of our reported $95 \%$ CIs are suggestive of favorable trends. Another limitation of this work is that PD-L1 status was not available in most of our melanoma and NSCLC patients, and therefore, was not included in our model. While PD-L1 expression in the tumor is predictive of response to PD-1 blockade therapy in several cancers, PD-L1 is not routinely tested in melanoma or used to select patients for treatment, as even PD-L1-negative patients may respond. ${ }^{329}$ Alternatively, we collected tumor mutation burden data that were available for a subset of patients that underwent genomic testing (Foundation Medicine), but the limited available data were insufficient for inclusion in multivariate analysis. Currently, it remains unclear how tumor mutation burden impacts melanoma in regards to predicting response to ICI therapies. ${ }^{30}$

\section{CONCLUSIONS}

In conclusion, our study showed that concomitant COXi use during ICI therapy for patients with metastatic melanoma and NSCLC is associated with dramatic improvements in outcomes. This analysis is, to our knowledge, the first report of the beneficial effect of COXi use in patients receiving ICIs. We also found an intriguing interaction between NLR and COXi use on TTP in patients with melanoma, suggesting the potential role of COXi to reverse immune tolerance in cancer patients. Our findings provide clinical evidence to support the underlying mechanism of synergy between ICI and COXi through reversing the immunosuppressive state of tumor microenvironment. These findings merit prospective study to evaluate this low-cost and easily implementable strategy that has potential to significantly improve the outcomes of patients receiving ICI.

\section{Author affiliations}

${ }^{1}$ Radiation Oncology, Rutgers Cancer Institute of New Jersey, New Brunswick, New Jersey, USA

${ }^{2}$ Radiation Oncology, Ohio State University Comprehensive Cancer Center, James Cancer Hospital and Solove Research Institute, Columbus, $\mathrm{OH}$, United States

${ }^{3}$ Biometrics Division, Rutgers Cancer Institute of New Jersey, New Brunswick, New Jersey, USA

${ }^{4}$ Radiation Oncology, University of Pennsylvania, Philadelphia, Pennsylvania, USA ${ }^{5}$ Medical Oncology, Rutgers Cancer Institute of New Jersey, New Brunswick, New Jersey, USA

${ }^{6}$ Radiology, Rutgers Cancer Institute of New Jersey, New Brunswick, New Jersey, USA

${ }^{7}$ Medical Oncology, Dana Farber Cancer Institute, Boston, Massachusetts, USA

${ }^{8}$ Merck \& Co Inc, Rahway, New Jersey, USA

${ }^{9}$ Medical Oncology, New York University School of Medicine, New York, New York, USA

Contributors S-JW contributed to study conceptualization, data curation, formal analysis, methodology, and the majority of the manuscript writing, review and editing. KK contributed to data curation, formal analysis, and manuscript writing. SK and NY-R contributed to formal statistical analysis, methodology, and manuscript writing. SHC and JLN contributed to formal radiology review of patient imaging per the RECIST version 1.1 criteria. JM and RG collected tumor mutation burden data. AWS and MAG contributed to manuscript review and editing. JMM contributed to data curation and project administration and supervision. SKJ contributed to study conceptualization, formal analysis, project administration, supervision, and manuscript review and editing. SKJ is guarantor. All authors read and approved the final manuscript.

Funding The authors have not declared a specific grant for this research from any funding agency in the public, commercial or not-for-profit sectors.

Competing interests None declared.

Patient consent for publication Not required.

Ethics approval This study has been approved by the Rutgers Institutional Review Board (Study \# Pro2018002434).

Provenance and peer review Not commissioned; externally peer reviewed.

Data availability statement Data are available upon reasonable request. The datasets generated and analyzed during the current study are not publicly available due to privacy of patients' protected health information under the Health Insurance Portability Accountability Act.

Open access This is an open access article distributed in accordance with the Creative Commons Attribution Non Commercial (CC BY-NC 4.0) license, which permits others to distribute, remix, adapt, build upon this work non-commercially, and license their derivative works on different terms, provided the original work is properly cited, appropriate credit is given, any changes made indicated, and the use is non-commercial. See http://creativecommons.org/licenses/by-nc/4.0/.

\section{ORCID iDs}

Shang-Jui Wang http://orcid.org/0000-0001-6166-2807

Roman Groisberg http://orcid.org/0000-0001-8970-7675

Ann W Silk http://orcid.org/0000-0003-3877-3984

\section{REFERENCES}

1 Hodi FS, O'Day SJ, McDermott DF, et al. Improved survival with ipilimumab in patients with metastatic melanoma. $N$ Engl J Med 2010;363:711-23.

2 Topalian SL, Sznol M, McDermott DF, et al. Survival, durable tumor remission, and long-term safety in patients with advanced melanoma receiving nivolumab. J Clin Oncol 2014;32:1020-30.

3 Larkin J, Chiarion-Sileni V, Gonzalez R, et al. Combined nivolumab and ipilimumab or monotherapy in untreated melanoma. $N$ Engl J Med 2015;373:23-34. 
4 Wolchok JD, Chiarion-Sileni V, Gonzalez R, et al. Overall survival with combined nivolumab and ipilimumab in advanced melanoma. $\mathrm{N}$ Engl J Med 2017;377:1345-56.

5 Reck M, Rodríguez-Abreu D, Robinson AG, et al. Pembrolizumab versus chemotherapy for PD-L1-positive non-small-cell lung cancer. N Engl J Med 2016;375:1823-33.

6 Gandhi L, Rodríguez-Abreu D, Gadgeel S, et al. Pembrolizumab plus chemotherapy in metastatic non-small-cell lung cancer. $N$ Engl J Med 2018;378:2078-92.

7 Mok TSK, Wu Y-L, Kudaba I, et al. Pembrolizumab versus chemotherapy for previously untreated, PD-L1-expressing, locally advanced or metastatic non-small-cell lung cancer (KEYNOTE-042): a randomised, open-label, controlled, phase 3 trial. Lancet 2019;393:1819-30.

8 Reck M, Rodríguez-Abreu D, Robinson AG, et al. Updated Analysis of KEYNOTE-024: Pembrolizumab Versus Platinum-Based Chemotherapy for Advanced Non-Small-Cell Lung Cancer With PD-L1 Tumor Proportion Score of $50 \%$ or Greater. J Clin Oncol 2019;37:537-46.

9 Wang D, Dubois RN. Eicosanoids and cancer. Nat Rev Cancer 2010;10:181-93.

10 Lee C, Ramirez JA, Guitart J, et al. Expression of cyclooxygenase-2 and peroxisome proliferator-activated receptor gamma during malignant melanoma progression. J Cutan Pathol 2008;35:989-94.

11 Chan AT, Ogino S, Fuchs CS. Aspirin and the risk of colorectal cancer in relation to the expression of COX-2. N Engl J Med 2007;356:2131-42.

12 Chan AT, Ogino S, Fuchs CS. Aspirin use and survival after diagnosis of colorectal cancer. JAMA 2009;302:649-58.

13 Hamada T, Cao Y, Qian ZR, et al. Aspirin use and colorectal cancer survival according to tumor CD274 (programmed cell death 1 ligand 1) expression status. J Clin Oncol 2017;35:1836-44.

14 Rothwell PM, Wilson M, Price JF, et al. Effect of daily aspirin on risk of cancer metastasis: a study of incident cancers during randomised controlled trials. Lancet 2012;379:1591-601.

$15 \mathrm{Ma} \mathrm{J}$, Cai Z, Wei H, et al. The anti-tumor effect of aspirin: what we know and what we expect. Biomed Pharmacother 2017;95:656-61.

16 Zelenay S, van der Veen AG, Böttcher JP, et al. CyclooxygenaseDependent tumor growth through evasion of immunity. Cell 2015;162:1257-70.

17 Böttcher JP, Bonavita E, Chakravarty P, et al. Nk cells stimulate recruitment of cdc1 into the tumor microenvironment promoting cancer immune control. Cell 2018;172:1022-37.
18 Botti G, Fratangelo F, Cerrone M, et al. Cox-2 expression positively correlates with PD-L1 expression in human melanoma cells. J Trans/ Med 2017;15:46

19 Hamy A-S, Tury S, Wang X, et al. Celecoxib with neoadjuvant chemotherapy for breast cancer might worsen outcomes differentially by COX-2 expression and ER status: exploratory analysis of the REMAGUS02 trial. J Clin Oncol 2019;37:624-35.

20 Edelman MJ, Watson D, Wang $X$, et al. Eicosanoid modulation in advanced lung cancer: cyclooxygenase-2 expression is a positive predictive factor for celecoxib + chemotherapy--Cancer and Leukemia Group B Trial 30203. J Clin Oncol 2008;26:848-55.

21 Edelman MJ, Wang X, Hodgson L, et al. Phase III randomized, placebo-controlled, double-blind trial of celecoxib in addition to standard chemotherapy for advanced non-small-cell lung cancer with cyclooxygenase-2 overexpression: CALGB 30801 (Alliance). J Clin Oncol 2017;35:2184-92.

22 Templeton AJ, McNamara MG, Šeruga B, et al. Prognostic role of neutrophil-to-lymphocyte ratio in solid tumors: a systematic review and meta-analysis. J Natl Cancer Inst 2014;106:dju124.

23 Shaul ME, Fridlender ZG. Tumour-Associated neutrophils in patients with cancer. Nat Rev Clin Oncol 2019;16:601-20.

24 Maymani $\mathrm{H}$, Hess K, Groisberg R, et al. Predicting outcomes in patients with advanced non-small cell lung cancer enrolled in early phase immunotherapy trials. Lung Cancer 2018;120:137-41.

25 Ferrucci PF, Ascierto PA, Pigozzo J, et al. Baseline neutrophils and derived neutrophil-to-lymphocyte ratio: prognostic relevance in metastatic melanoma patients receiving ipilimumab. Ann Oncol 2016;27:732-8

26 Mezquita L, Auclin E, Ferrara R, et al. Association of the lung immune prognostic index with immune checkpoint inhibitor outcomes in patients with advanced non-small cell lung cancer. JAMA Oncol 2018;4:351-7.

27 Park W, Lopes G. Perspectives: neutrophil-to-lymphocyte ratio as a potential biomarker in immune checkpoint inhibitor for non-small-cell lung cancer. Clin Lung Cancer 2019;20:143-7.

28 Wilky BA. Immune checkpoint inhibitors: the linchpins of modern immunotherapy. Immunol Rev 2019;290:6-23.

29 Mandalà M, Merelli B, Massi D. Pd-L1 in melanoma: facts and myths. Melanoma Manag 2016;3:187-94.

30 Twitty CG, Huppert LA, Daud Al. Prognostic biomarkers for melanoma immunotherapy. Curr Oncol Rep 2020;22:25. 